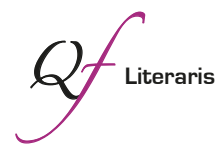

\title{
Cartas de Margarita de Parma (1578-1581) en la edición de Charles Piot
}

\author{
Letters by Margarita de Parma (1578-1581) \\ in Charles Piot's edition
}

\section{M. ${ }^{a}$ Antonia Blat Mir}

Florida State University International Programs.mblat@fsu.edu Recibido: 23/04/2017. Aceptado: 03/10/2017

Resumen: Estudio sobre la edición llevada a cabo a mediados del siglo XIX sobre la correspondencia de Margarita de Parma. La collatio de los documentos impresos con los originales de la correspondencia entre Margarita de Parma, hija natural de Carlos V, y el cardenal Granvela, secretario en este momento (1578-1581) del rey Felipe II, con los volúmenes editados por Charles Piot y conservados en la Bibliothèque Municipale de Besançon, y en el Archivio di Stato di Napoli, arrojan luz sobre la metodología usada en la edición de las cartas y hace necesaria una revisión del epistolario.

Palabras clave: Margarita de Parma; Granvela; Países Bajos; Correspondencia; Siglo XVI.

\begin{abstract}
Study about the edition, done in mid 19th century, of the Margaret of Parma's correspondence. The collatio of the documents printed with the original ones of this correspondance between Margaret of Parma, illegitimate daughter of Charles V and the cardinal Antonine Perrenot de Granvelle, secretary in this period (1578-1581) of Philip II, with the volumes edited by Charles Piot kept in the Bibliothèque Municipale of Besançon, and in the Archivio di Stato of Naples, show worthy information about the methodology used in the edition of the letters and make necessary a revision of the collection itself.
\end{abstract}

Keywords: Margaret of Parma; Granvelle; Netherlands; Correspondance; 16th century. ción de Charles Piot”. Quaderns de Filologia: Estudis Literaris 22: 27-39. doi: 10.7203/qfed.22.11193 



\section{Introducción}

Ante la edición del epistolario de Margarita de Parma, es necesario revisar el trabajo de Charles Piot, historiador, que llevó a cabo la edición de las cartas, conservadas en la Bibliothèque Municipale de Besançon y en el Archivio di Stato de Nápoles y relativas al periodo comprendido entre los años 1565-1583. Los tomos analizados en este artículo son los números 7 y 8 , entre los años 1578 y 1579 en el tomo 7, y los dos años siguientes, 1580 y 1581 , en el volumen $8^{1}$.

El porqué de la importancia de estos años lo encontramos en el prefacio del propio editor en el que resume los acontecimientos históricos, políticos e incluso privados o familiares del rey Felipe II, su hermana Margarita de Austria y su sobrino Alejandro Farnese.

El volumen 7 refleja los acontecimientos desde el 5 de enero de 1578 hasta el 9 de diciembre de 1578. En el volumen 8 las cartas empiezan el 3 de enero de 1580 y finaliza con una carta del cardenal Granvela a Margarita de Parma, datada el 22 de diciembre de 1581.

Los criterios de edición explícitos se limitan a presentar en el prefacio de cada tomo el número de cartas recogidas, los años que comprenden y los nombres de los personajes que mantenían correspondencia con el cardenal.

El volumen 7 contiene 186 cartas desde 1578 hasta 1579 sin contar las del Apéndice. Hay 63 cartas del cardenal Granvela a Margarita de Parma y 1 de Margarita al cardenal. También hay una del rey Felipe II a su hermana Margarita. El resto de las cartas están dirigidas al rey Felipe II, a don Juan de Austria, su hermanastro, a Alejandro Farnese, príncipe de Parma, al duque d'Aesrshot, a Emmanuele Filiberto de Saboya, al duque de Toscana, al duque de Urbino, al cardenal Farnese, a François

\footnotetext{
* Esta investigación se inscribe en el proyecto Las mujeres en la casa de Austria (15261567). Corpus Documental (Ref. FFI2014-52227-P), concedido por el ministerio de Economía y Competitividad, y dirigido por la Dra. Júlia Benavent.

${ }^{1}$ En 1839 un decreto real del 5 de marzo confiere a Piot el título de conservador adjunto honorario de la Biblioteca Real y quedará a cargo de la dirección del gabinete de medallas y monedas. El autor de esta edición es también archivero general del reino y miembro de la academia real de las ciencias, de las letras y de las bellas artes de Bélgica, y miembro de la comisión real de historia. Edita junto a M. Gachard los documentos de varios manuscritos sobre la historia de los Países Bajos y continúa su labor en solitario en los tomos 7 y 8 de la correspondencia del Cardenal Granvela sobre las cartas conservadas en Besançon, Simancas y, en su mayoría, el Archivio di Stato de Nápoles.
} 
de Vergy, conde de Champlitte, al consejero Fonck, a Antonio Pérez y al prior de Bellefontaine. En el Apéndice hay 92 piezas en las que se explican los acontecimientos más relevantes que se mencionan en las cartas: sobre la complicada situación de los negocios en los Países Bajos, los asuntos privados, etc. La gran preocupación de Felipe II en ese momento era la paz en los Países Bajos sin recurrir a las armas. El apéndice, en cambio, se dedica a recoger cartas cuyo contenido puede ayudar a arrojar luz sobre los acontecimientos mencionados en la correspondencia del cardenal. El resto del prefacio se dedica a la explicación de la historia de los Países Bajos, avalada por los manuscritos editados.

El tomo 8 contiene 154 cartas de los años 1580 y 1581 . En el apéndice hay 73 cartas más con memorias, informes y otras piezas que sirven para explicar los hechos a los que se alude en los manuscritos del cuerpo principal. Entre ellas hay 60 cartas de Granvela a Margarita de Parma y 40 de la misma Margarita al cardenal Granvela. Otros personajes de la época que aparecen como emisores o destinatarios de las misivas son el rey Felipe II, Alejandro Farnese, el duque de Toscana, el duque de Medina Sidonia, Filiberto duque de Saboya, d'Assonleville, el conde Pierre-Ernst de Mansfeld, Richardot, Morillon, el prior de Belle-Fontaine, don Juan Idiaquez, el duque de Aerschot, Thomas Myller y el abad de Maroille.

Desde el punto de vista del contenido, se constatan los razonamientos de Granvela a Margarita para que asumiera el gobierno de los Países Bajos y también sobre otros hechos personales de la duquesa como los enredos con el duque de Toscana contra los intereses de Margarita, viuda de Alejandro de Medicis, su primer marido; los asuntos relacionados con la conspiración de Claudio Landi contra su segundo marido, Octavio Farnese; las pretensiones de su nieto, Ranuccio Farnese, al trono de Portugal; el matrimonio de su nieta Margarita; el futuro de su sobrina, la hija de don Juan de Austria, de quien intentan deshacerse al mandarla a un convento, etc.

En conclusión, observamos que el argumento principal es siempre el gobierno de los Países Bajos que es el centro de interés, en el momento del encargo de esta obra, del gobierno belga. También encontramos algunas cartas con temas personales como la preocupación de Margarita por la salud de su hermano y su desconfianza hacia los médicos, o su tristeza tras la muerte de la reina el 26 de octubre de 1580, quedando 
puntualmente informada de los avances del rey gracias al cardenal (carta del 16 de octubre 1580).

Cada tomo, tras la presentación de las cartas editadas, continúa, como hemos dicho, con un apéndice de cartas temáticamente relacionadas, una tabla cronológica de los documentos contenidos en el volumen -indicando emisor y destinatario, lugar y fecha-, y una tabla alfabética de materias y personas.

\section{Marco histórico}

El periodo que comprenden estos dos volúmenes representa unos años tristemente significativos para la duquesa de Parma, tanto desde el punto de vista político como personal y familiar.

El gobierno de don Juan de Austria en los Países Bajos había desembocado en una explosión general revolucionaria en 1576. Antes de su muerte, don Juan designa como su sucesor a Alejandro de Parma, hijo de Margarita y de Octavio Farnese, nacido en Roma en 1544 y educado con don Carlos y don Juan en la corte de Felipe II. Tras la muerte de don Juan en 1578, el rey Felipe II cree que es necesario un nuevo tipo de gobierno. Entre las novedades se encuentra la repartición bicéfala del poder entre Margarita de Parma, su hermana natural, y el hijo de esta, Alejandro Farnese. A Margarita quiso encargarle las competencias civiles y ceder a su sobrino Alejandro las competencias militares partiendo de la idea de que madre e hijo se llevarían bien. La noticia de la muerte de don Juan le llega a Margarita en una carta de Granvela del 13 de octubre de 1578.

Desde un primer momento, el príncipe Farnese se negó a la repartición de poderes porque creía que atentaba contra su dignidad, insistiendo en que dejaría los Países Bajos cuando se cumplieran los seis meses de su mandato interino, como preveía el Tratado de Arras firmado entre los rebeldes y el Consejo de Estado en Mons el 17 de mayo de 1579. Por ello, una de las preocupaciones de Granvela era la conciliación de los intereses opuestos de Margarita y su hijo.

Por su parte, la duquesa de Parma había insistido reiteradamente en la necesidad de contar con una confirmación escrita oficial por parte de Felipe II, antes de iniciar su viaje a los Países Bajos, del cargo y los poderes que debía cubrir su misión. Para todo ello, la correspondencia con el cardenal Granvela se intensificó y, en ausencia de la claridad exigida 
por Margarita por parte de su hermano, se trataron otros temas políticos de gran relevancia.

No debemos olvidar que esta intensa comunicación llega tras un periodo convulso: durante el primer periodo de Margarita de Austria como gobernante de los Países Bajos (1559-1567), la intervención de la hija de Carlos V, contando con el apoyo de Guillermo de Orange, desembocó en la destitución del cardenal Granvela de su cargo como presidente del consejo de Estado (1556-1563), y el abandono de este de la corte flamenca.

Tras su marcha, Margarita continúa su campaña y se apresura a limitar la influencia de los "cardenalistas" en la corte para de este modo apaciguar los ánimos del pueblo. Así pues, el cardenal Granvela se recluye en Besançon, hasta que más tarde es enviado a Roma desde donde escribe todas las cartas que encontramos en el Volumen 7 de esta edición, exactamente hasta el 25 de mayo de 1579.

Pero en la época que nos ocupa, y con el retorno de Granvela a su función de consejero del rey, la correspondencia entre ambos se retoma de modo frecuente. De hecho, es el propio cardenal quien influye en la expulsión del archiduque Mathias de Austria de Bruselas por ser mal consejero para el rey e insiste en poner en su lugar a la princesa Margarita como se ve en una carta de marzo de 1578. Granvela apoya siempre la decisión del rey de mantener a madre e hijo en el gobierno con la repartición de poderes e intenta convencerlos con cada misiva de que es la mejor opción. Incluso se compromete en primera persona a asegurarse de que las provisiones lleguen siempre a tiempo. Tanto es así que Granvela muestra su preocupación en los consejos que continuamente transmite a Margarita sobre el comportamiento que deberá tener: deberá sospechar de los personajes de doble cara que siembran la descofianza bajo el pretexto de ser buenos servidores del rey.

Alejandro había sido confirmado en el tratado de Arras, de reconciliación con las provincias valonas, como sucesor de don Juan de Austria, con el cargo de gobernador y capitán general, a la espera de la llegada de un nuevo gobernador general, y solo como cargo temporal de seis meses, hasta el 31 de octubre de 1580, cuando debería dejar el puesto a otro príncipe o princesa de sangre real, si no, el poder pasaría al nuevo consejo de Estado constituido.

Las motivaciones del rey Felipe II para nombrar por segunda vez a Margarita gobernante de los Países Bajos son, entre otras, porque esta 
conocía la lengua de los países flamencos y franceses y también porque representaba la reconciliación y la clemencia más que la represión y la guerra. De todas formas no debemos pensar que Margarita de Parma no fue una estricta gobernante ya que, como vemos en algunas cartas de 1581 , le recuerda a Granvela que hay que actuar con firmeza. Por todo ello, Granvela intenta convencer a Margarita de que debe convertirse en consejera de su hijo ya que teme que el joven se deje llevar por la imprudencia. Así pues, una de sus misiones será la de actuar como mediadora entre los vencedores y los vencidos: este rol decisivo se confirma en el número de cartas dirigidas a la duquesa, muy superior al número de cartas enviadas a su hijo por parte del cardenal.

Granvela comunica a Margarita que se deberá establecer en Namur, una de las pocas provincias que quedaba en manos del gobierno de Felipe II, y ponerse en contacto con su hijo. Le encomienda cuestiones religiosas como establecer "todas las cosas como en tiempos de Carlos V" y mantener inviolable la religión católica. No quisieron ver que el problema no era la religión sino que los flamencos estaban en contra de los españoles por cómo los habían tratado y la imposición de la religión no iba a solucionarlo. Las cavilaciones de Margarita, en cambio, eran que la definición de su cargo seguía sin quedar clara y el silencio de su hermano comenzaba a hacer que ella misma dudara de que éste cumpliría con la prerrogativa de restituirle el castillo de Piacenza a los Farnese que había quedado en manos de los españoles desde 1556 y que, después de tantos años de negociaciones, Margarita veía alejarse de nuevo.

En la carta del 6 de enero de 1580 dice que está preparada para partir pero el inicio del viaje no se concreta hasta el mes de febrero, aun sin haber confirmado sus nuevos poderes en su antigua corte, con graves problemas de salud debido a la gota que sufría y sin haber concertado su viaje con su marido Octavio Farnese. Se lleva con ella a su nieta Margarita de 13 años, dejando a su otro nieto, Ranuccio Farnese de 11 años, a cargo de su marido Octavio Farnese. El viaje no resulta fácil desde el punto de vista físico, ni seguro: al llegar a Borgoña, deben parar por una indisposición de su nieta que ha contraído roséola. La peligrosidad por el posible encuentro de rebeldes en el camino hace que su hijo Alejandro le aconseje parar en Luxemburgo, una de las provincias que quedaron bajo la tutela del rey Felipe II tras el armisticio flamenco.

A principios de junio de 1580 Margarita parte de Besançon hacia los Países Bajos. Al llegar a final de mes a Luxemburgo, habría querido 
abrazar a su hijo pero Alejandro al ver la toma de posición de su madre, tan alejada de la suya, prefirió no encontrarse con ella y Margarita se quedó en Mons.

Finalmente el séquito real llega a Namur el 26 de julio en medio de un motín. En una carta a Granvela, Margarita defiende la posición de su hijo porque dice que la repartición del poder gubernamental sería inejecutable. Cinco meses después el rey manda una carta a ambos confirmando su mandato de repartición de poderes y les exige que obedezcan sin más.

Finalmente, Felipe II claudica ante la insistente presión de Alejandro y acepta confiarle todas las funciones de gobernador y capitán general sin límite de tiempo pero le pide a Margarita que permanezca junto a él. Ella permanece en Namur por afecto a los flamencos durante más de un año sin ningún cargo. Cuando finalmente llega la carta de confirmación del fin de su misión el 25 de julio de 1583, deja por última vez su tierra natal y llega a Italia el 14 de septiembre del mismo año. Madre e hijo no se volvieron a ver nunca más.

Pero el viaje de Margarita no había sido completamente inútil porque la intensa correspondencia con el cardenal Granvela sirvió para tratar asuntos importantes del mundo español y sobre todo de Italia. Por ejemplo de la posición del Franco Condado, que a Margarita le parecía de gran importancia estratégica por estar situada en lugar de pasaje entre Italia y Países Bajos. Los escritos de Margarita, que envía durante 1581 a España, contienen numerosas sugerencias sobre los desórdenes y la corrupción de la justicia en esta zona. También había observado abusos en la organización de abadías, conventos ya que esta era una provincia vulnerable por estar alejada y aislada. Entre las medidas sugeridas por la duquesa de Parma para asegurar la seguridad del condado de Borgoña están el pago de dinero a las tropas y la previsión de fondos para la mejora de las instituciones. También sugiere que la solución es "nombrar buenos ministros, justos, hábiles, imparciales y desinteresados".

\section{La edición}

La correspondencia editada por Piot, como continuación de la iniciada por Gachard, plantea una serie de interrogantes que derivan de la selección de las cartas editadas. Las cuestiones en primer lugar se refieren a la identificación de los documentos en el Archivio di Stato di Napoli 
$\mathrm{y}$ en la Bibliothèque Municipale de Besançon. No obstante, en la selección fueron descartadas algunas cartas que consideramos necesarias para comprender el desarrollo de los acontecimientos y de las respuestas políticas, construidas entre Granvela y Margarita mientras el rey Felipe estaba en Portugal. Esta correspondencia necesita ser completada para mejor conocimiento de lo que sucedió y para comprender el papel que llevó a cabo Margarita en el gobierno de los Países Bajos, mientras defendía los intereses de su hijo y de su marido en la recuperación de Parma.

No queda claro si Piot visitó personalmente el Archivio di Stato di Napoli porque no da más información que el número de legajo en que se encuentra cada carta pero no especifica el número de folio ni tampoco evidencia la división del recto y el verso. Aunque este último podría ser un criterio de edición porque es una información que tampoco se encuentra sobre las cartas conservadas en la Bibliothèque Municipale de Besançon (de las que sí ofrece dicha información).

En el tomo 7 hay un total de 64 cartas entre Granvela y Margarita. De ellas, todas excepto una, la última, es emisor el cardenal Granvela y van dirigidas a la duquesa de Parma. Por este motivo, la lengua de las 63 cartas de Granvela a Margarita es el francés, y solo encontramos una carta en italiano que corresponde a la carta número 183 del tomo. Esta será la única carta traducida en este volumen de entre las cartas analizadas.

En el tomo 8 en cambio, la correspondencia es recíproca y encontramos alternativamente cartas en francés y en italiano. De un total de 101 cartas entre los protagonistas, 42 tienen como emisora a Margarita y el resto (59) al cardenal Granvela. Ese será pues el número de cartas en italiano y en francés respectivamente. Las cartas en lengua italiana son objeto de traducción al francés por parte de Piot una vez más, pero observamos que no siempre sigue el mismo proceder, ya que en la mayoría de los casos se limita a hacer un resumen del contenido de las mismas y no una traducción.

\subsection{La collatio}

Los criterios de edición que encontramos son formales, como por ejemplo no marcar la transición con doble barra -ni de ninguna otra manera- entre el recto y el verso de un folio ni tampoco entre un folio y el 
siguiente para presentar de manera unificada la carta transcrita. No se hace pues, referencia a la foliación.

Por otro lado, tampoco presenta información del material físico, como las medidas de los originales, las marcas de agua, el estado en el que se encontraba la carta, etc.

Tampoco se incluye ninguna información ajena al contenido, lo que confirma su interés exclusivo por los manuscritos como fuente de información histórica. Hablamos por ejemplo de glosas marginales, comentarios, aclaraciones, explicaciones, puntualizaciones, etc. Por ejemplo, en el manuscrito original de la Bibliothèque Municipale de Besançon: Memorias del Cardenal de Granvela, t. XXXI, fol. 41-42, aparece el sintagma "la longhezza" en interlineado y en la edición de Piot se inserta en la transcripción sin niguna aclaración. Otro ejemplo de este tipo muestra en los fol. 58 a 59 del mismo tomo, una palabra tachada, por una confusión en la escritura y, a continuación, la palabra completa correcta: spesan speranza. Tampoco en este caso la transcripción ofrece ninguna referencia.

En algunos casos se desarrollan todas las abreviaturas de tratamiento $y$ en otras cartas no, lo que hace pensar en una cierta incoherencia en los criterios de edición. En cuanto a otras abreviaturas, encontramos los casos de boniss ${ }^{a}$, que aparece en el manuscrito como bonissima, o racc.to, que significa raccomandato. Pero el caso de fig.lo transcrito incorrectamente como figlio en más de una ocasión -se refiere a figliuolo-, parece indicar un origen no italiano del escribano, quizás hispánico.

En cuanto a la acentuación, Piot la añade pero no de forma sistemática y no en todas las palabras por igual. Aparecen palabras acentuadas que no presentan este signo de escritura en el manuscrito como por ejemplo: più, è, sanità, darà, prosperità, volontà, accertarà, riuscirà, malignità, cesserà, etc. Pero en otros casos de verbo en futuro también o de aguda que termina en "a", el editor decide no añadir la tilde: servira, prosperita, potra. Otro caso de fluctuación del acento son: puo y puó. Tampoco acentúa perche ni se como pronombre tónico. En otros casos, en los que sí aparece la palabra acentuada en el original, se toma la decisión de eliminarla en la versión editada (ejemplo: sta), lo que podría corresponder a un criterio de modernización de la acentuación del texto pero en seguida debemos abandonar esta hipótesis por la aparición de casos en los que mantiene la acentuación de palabras que hoy en día no se acentuarían como en hò. 
También encontramos oscilaciones en el uso de algunas grafías. Un ejemplo podría ser el sustantivo negotij, presentado hasta en dos ocasiones con esta forma - lo que confirma que no es un descuido- pero en una tercera aparición en la misma carta, se transcribe como negotii. Otra grafía usada por decisión del editor es la "ç" como transcripción de otras posibles como la "z", o la "c" en los casos siguientes: sença, benefiçio o cominçiato. Esta decisión podría reconducirnos de nuevo sobre la posibilidad de que el autor fuera hispanista.

La confusión entre la unión o separación de palabras también queda representada en el caso de instimabile que aparece en el manuscrito separado in stimabile.

A nivel sintáctico, también encontramos la incorporación en la transcripción de la preposición "da" como introductor de un complemento agente que en el original no aparece.

En numerosos casos encontramos inexactitudes de transcripción

\begin{tabular}{|l|l|}
\hline ORIGINAL & TRANSCRIPCIÓN \\
\hline si trova & se trova \\
\hline la servitu & lo servitio \\
\hline accettato & accettata \\
\hline poi che & per che \\
\hline bono pensiero & bone pensiero \\
\hline il & el \\
\hline si & se \\
\hline si trovasse & si trovassi \\
\hline forzata & forsata \\
\hline dua & duo \\
\hline A fare opera che & A fare per ché \\
\hline mie & mei \\
\hline haveva & havera \\
\hline dalla & della \\
\hline Vallone & Wallone \\
\hline effetto & effecto \\
\hline
\end{tabular}


En algunos casos parece que el cambio se deba a la voluntad de concordar en género o número las palabras como en:

\begin{tabular}{|l|l|}
\hline ORIGINAL & TRANSCRIPCIÓN \\
\hline mandati & $\begin{array}{l}\text { mandate (hace concordan- } \\
\text { cia }- \text { le istruzioni-) }\end{array}$ \\
\hline Le gente & Le genti \\
\hline
\end{tabular}

Pero en otros sucede lo contrario y rompe la concordancia del original:

\begin{tabular}{|l|l|}
\hline ORIGINAL & TRANSCRIPCIÓN \\
\hline Santa mano & Santo mano \\
\hline Ill $^{\text {ma }}$ & Ill $^{\mathrm{mo}}$ \\
\hline
\end{tabular}

La última de las particularidades que comentaremos es la decisión de no incluir la fecha ni la firma al pie de la carta. De hecho, el explicit para los índices muestra la última frase del cuerpo de la misma. O incluso a veces, unos puntos suspensivos que no solo sustituyen la fecha y firma de emisor, sino también las formales frases de cierre del lenguaje epistolar del siglo XVI. En uno de los casos, la transcripción termina con puntos suspensivos con nota a pie de página que informa de que falta una parte de la carta. En cambio en otro caso, la transcripción termina con un "etc." y al ver el original, descubrimos que falta toda una frase que quizás Piot no consideró importante.

\begin{tabular}{|l|l|}
\hline ORIGINAL & TRANSCRIPCIÓN \\
\hline $\begin{array}{l}\text {..vi metta la sua santo } \\
\text { mano. etc. }\end{array}$ & $\begin{array}{l}\text {.. vi metta la sua santa mano et a } \mathrm{V} \text {. S. Illma } \\
\text { conceda ogni felicita di Parma alli XVIII di } \\
\text { marzzo 1580. Di V. S. Ill }{ }^{\text {ma }} \text { et Parma }\end{array}$ \\
\hline
\end{tabular}

\section{Conclusión}

Ante la edición que pretendemos llevar a cabo sobre la correspondencia de Margarita de Parma, hemos analizado la que intercambiaron Margarita de Austria y el cardenal Antonio Perrenot de Granvela entre los años 
1578 y 1581. La correspondencia tiene un valor histórico indiscutible que aumentará cuando se reúna toda completa, no solo la relativa a los asuntos de Flandes. Por otra parte, la precisión y exactitud en la determinación de los criterios de edición, oscilantes e inseguros en la edición de Piot, permitirá revalorizar también desde el punto de vista filológico la edición de los documentos. La edición de Charles Piot continúa la obra de Gachard. Y nuestra pretensión es seguir lo que ellos iniciaron con mayor atención a los criterios de transcripción, y el rigor filológico.

\section{Bibliografía}

Fondos manuscritos:

Nápoles, Archivio di Stato di Napoli, Archivio Farnesiano.

Besançon, Bibliothèque Municipal de Besançon, Papiers d'Etat.

Literatura crítica:

Dumont, Georges-Henri. 1999. Marguerite de Parme. Bâtarde de Charles Quint. 1522-1586. Bruselas: Le Cri.

Gachard, Louis Prosper (ed.). 1867-1881. Correspondance de Marguerite d'Autriche. Bruselas: C. Muquardt.

Mantini, Silvia. 2003. Margherita d'Austria. Costruzioni politiche e diplomazia, tra corte farnese e monarchia spagnola. Roma: Bulzoni.

Piot, Charles (ed.). 1877-1896. Correspondance du Cardinal de Granvelle (1565-1586). Bruselas: F. Hayez. 
